\title{
CARACTERIZACIÓN GENÉTICA DE ACCESIONES SANMARTINENSES DEL BANCO NACIONAL DE GERMOPLASMA DE SACHA INCHI PIukenetia volubilis L. (E.E. EI Porvenir - INIA)
}

\author{
Mike CORAZON-GUIVIN ${ }^{1}$, Diana CASTRO-RUIZ ${ }^{2}$; Werner CHOTA-MACUYAMA ${ }^{2}$; Ángel \\ RODRÍGUEZ ${ }^{1}$, Danter CACHIQUE ${ }^{2}$, Emma MANCO ${ }^{4}$; Dennis DEL-CASTILLO ${ }^{2}$, Jean-François \\ RENNO $^{3}$, Carmen GARCÍA-DÁVILA ${ }^{2}$
}

1 Investigadores becados del Instituto de investigaciones de la Amazonía Peruana. Iquitos, Perú. E-mail: mikecorazon10@gmail.com

2 Instituto de Investigaciones de la Amazonía Peruana (IIAP). Laboratorio de Biología y Genética Molecular (LBGM). Apartado 784. Iquitos, Perú. E-mail: cdavila19@yahoo.com

3 Institut de Recherche pour le Développement (IRD). Montpellier, France.

4 Instituto Nacional de Investigación Agraria - INIA, San Martín, Estación El Porvenir, Tarapoto.

\section{RESUMEN}

El presente estudio analiza 12 accesiones sanmartinenses de Sacha Inchi Plukenetia volubilis del Banco Nacional de Germoplasma del INIA, utilizando la técnica molecular DALP. Para lo cual se colectaron muestras (Hojas) de un total de 24 plantas (dos por cada accesión), que fueron evaluadas utilizando los marcadores informativos (DALP221 y DALP233). El análisis de los perfiles genéticos entre las accesiones, mostró una alta diversidad con un total de 23 genotipos diferentes, observando que casi todas las accesiones analizadas presentaron dos genotipos, a excepción de los individuos Cub-02 y Ba.01-14 que compartieron un mismo genotipo. La planta SL-I-19 (accesión SL-I) mostró una fuerte divergencia genética, lo que sugiere la presencia de genotipos adicionales. Los métodos moleculares pueden hacer importantes contribuciones en la cuantificación de la diversidad genética de Sacha Inchi y otras especies de plantas en el Perú del Banco Nacional de Germoplasma. Este conocimiento es vital en la conservación y mejora genética de la especie.

PALABRAS CLAVE: Plukenetia volubilis, sacha inchi, diversidad genética, banco de germoplasma, DALP.

\section{GENETIC CHARACTERIZATION SACHA INCHI (Plukenetia volubilis) GERMPLASM ACCESSIONS FROM SAN MARTÍN, PERU}

\begin{abstract}
The present study examines 12 accessions of Sacha Inchi (Plukenetia volubilis) from San Martín of Peruvian National Germplasm Bank (INIA), using molecular techniques (DALP). Leaves were collected from a total of 24 individuals (two per accession), and evaluated using the informative markers DALP221 and DALP233. Genetic analysis showed high diversity between accessions, with a total of 23 different genotypes represented. In all but one case, the individuals of a given accession differed in genotype; only Cub-02 and Ba.01-14 shared a single genotype. Individual SL-I-19 showed strong genetic divergence, suggesting the presence of additional genotypes. Molecular methods can make important contributions in quantifying the genetic diversity of Sacha Inchi and other plant species in the Peruvian National Germplasm Bank. This knowledge is vital in the conservation and genetic improvement of the species.
\end{abstract}

KEYWORDS: Plukenetia volubilis, sacha inchi, Genetic diversity, Germplasm Bank, DALP. 


\section{INTRODUCCIÓN}

El sacha inchi Plukenetia volubilis L. representa un cultivo promisorio de gran importancia en la amazonia peruana (semillas con elevadas concentraciones de ácidos grasos esenciales tipo -3 y -6 (Hamaker et al., 1992). Se ha observado que esta especie presenta una amplia variabilidad tanto en su morfología, como en su capacidad de producción por planta y contenidos de aceite (Valles, 1990; Rodríguez et al., 2010). La estación experimental "El Porvenir" del Instituto Nacional de Investigación Agraria-INIA, con el intuito de caracterizar esta gran diversidad, viene implementando el Banco Nacional de Germoplasma de sacha inchi, el cual actualmente cuenta con aproximadamente 51 accesiones colectadas en diferentes departamentos de la amazonía peruana.

Los bancos de germoplasma, son herramientas para la conservación de especies silvestres, de las cuales aún conocemos poco, pero sabemos que encierran parte del pool genético de una determinada especie. La caracterización genética de un banco permite conocer el tipo de material genético que disponemos, así como identificar y caracterizar accesiones sobresalientes o en su defecto identificar duplicados (Bórem \& Caixeta, 2006). Además, el conociendo de los recursos genéticos permiten obtener nuevas variedades de plantas, a través del mejoramiento genético clásico o la ayuda de la biotecnología (Rossiter et al., 2000; Eirizik et al., 2001). La caracterización genética y detección de la diversidad genética, mediante técnicas moleculares ha sido muy utilizada en los últimos años, a su gran capacidad de detectar diferencias entre individuos que podrían estar cercanamente relacionados (Ferreira \& Gattapaglia, 1998). En este sentido, la Amplificación Directa de Polimorfismo de Longitud (DALP) es una técnica molecular que usa marcadores universales que puede ser aplicable para el estudio genético de diferentes especie de plantas y animales (Perrot-Minnot et al., 2000). Esta técnica fue utilizada por primera vez por Desmarais et al. en 1998, para diferenciar dos sub especies de ratones (Mus musculus musculus y M. musculus domesticus). Demostró ser altamente informativa para el estudio de diversidad genética de plantas (Ha et al., 2001; Yun-shu et al., 2008). Recientemente fue utilizada por Corazon-Guivin et al. (2008), para estudiar la variabilidad genética en cuatro poblaciones naturales de $P$. volubilis en la región de San Martín, obteniéndose resultados muy informativos sobre la diversidad y la estructura genética de cuatro poblaciones naturales (Tununtunumba, Shica, Cerro Alto y Habana).

En el caso del sacha inchi (nombre común utilizado para nominar una serie de especies diferentes, cuyos límites taxonómicos no son del todo conocidos), existe necesidad de caracterizar la diversidad genética encerrada en el banco de germoplasma de sacha inchi, para poder analizar cuál es el grado de diversidad genética que encierran las accesiones. En este sentido, el presente estudio tuvo como finalidad contribuir al conocimiento genético del sacha inchi, utilizando la técnica molecular DALP (Amplificación Directa de polimorfismo de Longitud), la cual es una poderosa herramienta para el estudio de la diversidad genética entre accesiones.

\section{MATERIALES Y MÉTODOS}

\section{COLECTA, CODIFICACIÓN Y CONSERVACIÓN DEL MATERIAL BIOLÓGICO}

La colecta de las muestras se realizó en el Banco Nacional de Germoplasma de sacha inchi del Instituto Nacional de Investigación Agraria INIA, ubicado en el distrito de Juan Guerra, departamento de San Martín.

Fue colectado tejido foliar de 24 plantas (2 plantas/accesión) pertenecientes a 12 accesiones sanmartinenses (Tabla1). El material fue acondicionado en forma individual en papel aluminio perfectamente codificado, dentro de tubos de $50 \mathrm{ml}$ con tapa rosca conteniendo un preservante sintético (sulfato de calcio anhidro), hasta el momento de la extracción de ADN en el Laboratorio de Biología y Genética Molecular (LBGM) del Instituto de Investigaciones de la Amazonía Peruana (IIAP), ubicada en el centro de investigaciones de Quistococha, carretera Iquitos-Nauta $\mathrm{km} \mathrm{4.5,} \mathrm{en} \mathrm{la}$ ciudad de Iquitos.

\section{EXTRACCIÓN Y AMPLIFICACIÓN DE ADN}

La extracción de ADN, fue realizada mediante el protocolo CTAB de Doyle \& Doyle (1987), a partir de $100 \mathrm{mg}$ de tejido foliar. La selección de los marcadores DALP (DALP 01 y DALP 04) para la caracterización genética del las accesiones fue realizada en base a información preliminar proporcionada por CorazónGuivin et al. (2008) sobre los marcadores más informativos de la técnica molecular DALP para esta especie (Tabla 2). La reacción de amplificación fue realizada en un volumen total de $25 \mu 1$, conteniendo 5 $\mathrm{U} / \mu 1$ de Taq polimerasa, $100 \mathrm{ng} / \mu \mathrm{l}$ de ADN molde, $5 \mathrm{X}$ de Buffer, 10 mM dNTPs, $25 \mathrm{mM}$ de $\mathrm{MgCl}_{2}, 10 \mu \mathrm{M}$ de cada primer y agua ultrapura. Las condiciones de temperatura fueron: denaturación inicial a $95{ }^{\circ} \mathrm{C} x$ $1 \mathrm{~min}$; seguida de 29 ciclos de: denaturación a $91{ }^{\circ} \mathrm{C} \mathrm{x}$ $30 \mathrm{~s}$, hibridación a $42.9^{\circ} \mathrm{C}$ x $30 \mathrm{~s}$, y extensión a $72{ }^{\circ} \mathrm{C} \mathrm{x}$ $30 \mathrm{~s}$; seguida de una extensión final a $72{ }^{\circ} \mathrm{C}$ x $5 \mathrm{~min}$. El patrón de bandas obtenido, fue verificado en geles de poliacrilamida al $6 \%$, teñido con nitrato de plata mediante el método Rabat (Sambrook \& Russell, 1991). 


\section{ANÁLISIS DEDATOS}

El polimorfismo entre las muestras fue analizado mediante la observación de presencia y ausencia de las bandas entre los individuos. A partir del cual se realizó una matriz binaria en base a la presencia (1) y ausencia (0) de las bandas diferenciales (polimorficas). El genotipo para cada uno de los individuos fue establecido a partir de la combinación de los perfiles de bandas generadas por cada uno de los tres primers DALP. La variabilidad genética fue establecida mediante el Análisis Factorial de Correspondencia (AFC). Este análisis fue realizado con la ayuda del Software GENETIX versión 4.05 (Belkhir et al., 2004).

Tabla 1. Código del banco, código nacional y procedencia de las accesiones de sacha inchi Plukenetia volubilis analizadas.

\begin{tabular}{c|c|c|c}
\hline $\mathbf{N}^{\mathbf{0}}$ & $\begin{array}{c}\text { CódIGO DE ENTRADA } \\
\text { BANCO }\end{array}$ & CÓDIGO NACIONAL & PROCEDENCIA \\
\hline 1 & BNOT001 & PER000394 & Shilcayo \\
\hline 2 & BNOT002 & PER000394 & Shilcayo \\
3 & BNOT003 & PER000395 & Pinto Recodo \\
\hline 5 & BNOT005 & PER000396 & Cumbaza \\
\hline 7 & BNOT032 & PER000416 & Chazuta \\
\hline 8 & BNOT034 & PER000418 & Moyobamba \\
\hline 11 & BNOT035 & PER000419 & San Fernando \\
\hline 12 & BNOT007 & PER000397 & Barranquita \\
\hline 13 & BNOT008 & PER000397 & Barranquita \\
\hline 14 & BNOT009 & PER000398 & Habana \\
\hline 37 & BNOT037 & PER000421 & Alto Shamboyacu \\
\hline 38 & BNOT038 & PER000422 & Alto Pucayacu \\
\hline
\end{tabular}

Tabla 2. Número y secuencia de los marcadores de la técnica DALP utilizados para caracterizar las accesiones de sacha inchi Plukenetia volubilis.

\begin{tabular}{c|c|c|c}
\hline TIPO DE PRIMER & PRIMERS & FUENTE & \multicolumn{1}{c}{ SECUENCIA } \\
\hline Primer reverso & DALP R & Desmarais et al. 1998 & TTTCACACAGGAAACAGCTATGAC \\
Primers selectivos & DALP221 & Desmarais et al. 1998 & GTTTTCCCAGTCACGACGC \\
& DALP233 & Desmarais et al. 1998 & GTTTTCCCAGTCACGACACG \\
\hline
\end{tabular}




\section{RESULTADOS Y DISCUSIÓN}

Hasta años atrás, los mejoradores echaban mano de técnica de caracterización morfológica para conocer los materiales genéticos existente en las colecciones; a partir de los datos de caracterización y validación se decidían sobre cuales accesiones debían ser incluidas en los programas de mejoramiento. Actualmente con el avance de los marcadores moleculares, los investigadores tienen una herramienta que puede auxiliarlos en la estimación de la diversidad genética existente en la colección, en las relaciones genéticas entre los materiales para definir la dirección de los cruzamientos y selección de progenitores, así como poder acompañar la introgresión de genes durante el proceso de mejoramiento (Borém \& Caixeta, 2006). La caracterización genética y detección de la diversidad genética, mediante técnicas moleculares ha sido muy utilizada en los últimos años, debido a su gran capacidad de detectar diferencias entre individuos que podrían estar cercanamente relacionados. En este sentido, la Amplificación Directa de Polimorfismo de Longitud (DALP) es una técnica molecular que usa marcadores universales que puede ser aplicable para el estudio genético de diferentes especie de plantas y animales (PerrotMinnot et al., 2000). Esta técnica fue utilizada por primera vez por Desmarais et al. en 1998, para diferenciar dos sub especies de ratones (Mus musculus musculus y M. musculus domesticus). Esta técnica demostró también ser altamente informativa para el estudio de diversidad genética de plantas (Ha et al., 2001; Yun-shu et al., 2008). Recientemente fue utilizada por Corazon-Guivin et al. (2008), para estudiar la variabilidad genética en cuatro poblaciones naturales de $P$. volubilis en la región de San Martín, obteniéndose resultados muy informativos sobre la diversidad y la estructura genética de cuatro poblaciones naturales (Tununtunumba, Shica, Cerro Alto y Habana).

Las 24 plantas (2 plantas/accesión) analizadas del Banco Nacional de Germoplasma del INIA, con los marcadores DALP221 (Figura 1) y DALP233 (Figura 2) generaron un total de 46 bandas de ADN, haciendo un promedio de 23 bandas por marcador usado (Tabla $3)$. De las 46 bandas producidas, solo 28 fueron informativas o polimorficas (60.87\%). Asimismo, se encontró un total de 23 genotipos diferentes (Tabla 4), observando que casi todas las accesiones analizadas presentaron genotipos distintos. Sin embargo, se pudo apreciar que el genotipo ABAAABBAABAAAAA BBABBBABABBAB es compartido por los individuos Cub-02 y Ba.01-14 de las accesiones Cumbaza y Barranquita 01 respectivamente. También se pudo observar que las dos plantas analizadas de cada accesión son diferentes a nivel genético. La presencia de una alta diversidad genética en las 12 accesiones sanmartinenses, hace que este material evaluado pueda ser considerado para futuros trabajos de mejoramiento genético, previa evaluación de su comportamiento a nivel de campo.

La tabla 4 muestra también que, el genotipo BBABBABABABBBBBBBBBBBBABABAA de la planta SL-I-19 (accesión SL-I) es genéticamente muy diferente de todas las plantas evaluadas. Esto podría deberse a que esta accesión contienen una fracción de información genética no contemplada por el reducido número de plantas analizadas o en su defecto a un error involuntario en el momento de nominar la accesión en campo.

La proyección gráfica (Figura 3) de los dos primeros factores muestra que las accesiones se encuentran distribuidas en forma irregular dentro de las dos agrupaciones principales. Las accesiones de Moyobamba, Pinto Recodo, Alto Shambuyacu, Barranquita-02 y Habana muestran sus dos individuos más relacionados con la agrupación $\mathrm{A}$, en tanto los individuos de la accesión San Fernando se encuentra relacionadas a la agrupación $\mathrm{B}$. El resto de accesiones (Shilcayo-01, Shilcayo-02, Cumbaza, SL-1 y Chazuta) presentan individuos en ambas agrupaciones. Así mismo se puede observar que uno de los individuos de la accesión SL-I presenta un perfil genético muy diferente del otro representante de la misma accesión y de las otras accesiones.

El conocimiento de la distribución de la diversidad genética de los cultivos, es de vital importancia cuando se pretenden desarrollar programas exitosos de mejoramiento y conservación de una especie. En el caso del sacha inchi se hace necesario tomar ventaja de la amplia diversidad genética encontrada en el banco de El Porvenir, a fin de contrarrestar la reducida diversidad reportada por Corazon-Guivin et al. (2008), para algunas poblaciones naturales de la región San Martín, a consecuencia de la fragmentación del habitad de estas poblaciones naturales, producto de una expansión agrícola constante. El conocimiento de la diversidad genética presente en las colecciones permitirá además a los mejoradores a través de metodologías adecuadas generar materiales genéticos superiores en un lapso de tiempo mucho mas corto que mediante evaluaciones morfológica. 

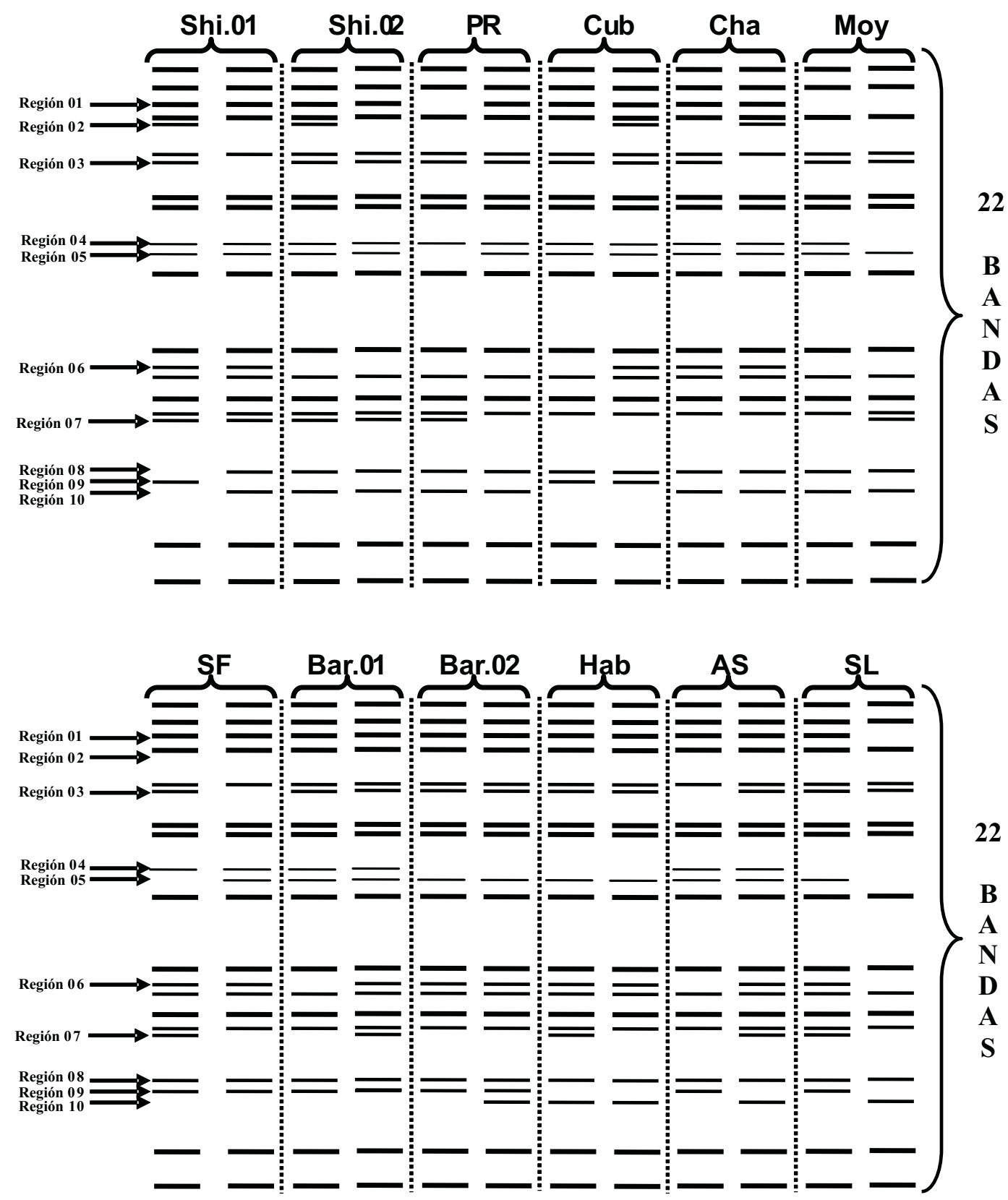

Figura 1. Representación gráfica del gel de poliacrilamida $6 \%$ mostrando los fragmentos de ADN y las regiones informativas amplificadas con el marcador DALP221 en las accesiones de sacha inchi Plukenetia volubilis del Banco de Germoplasma del INIA. 

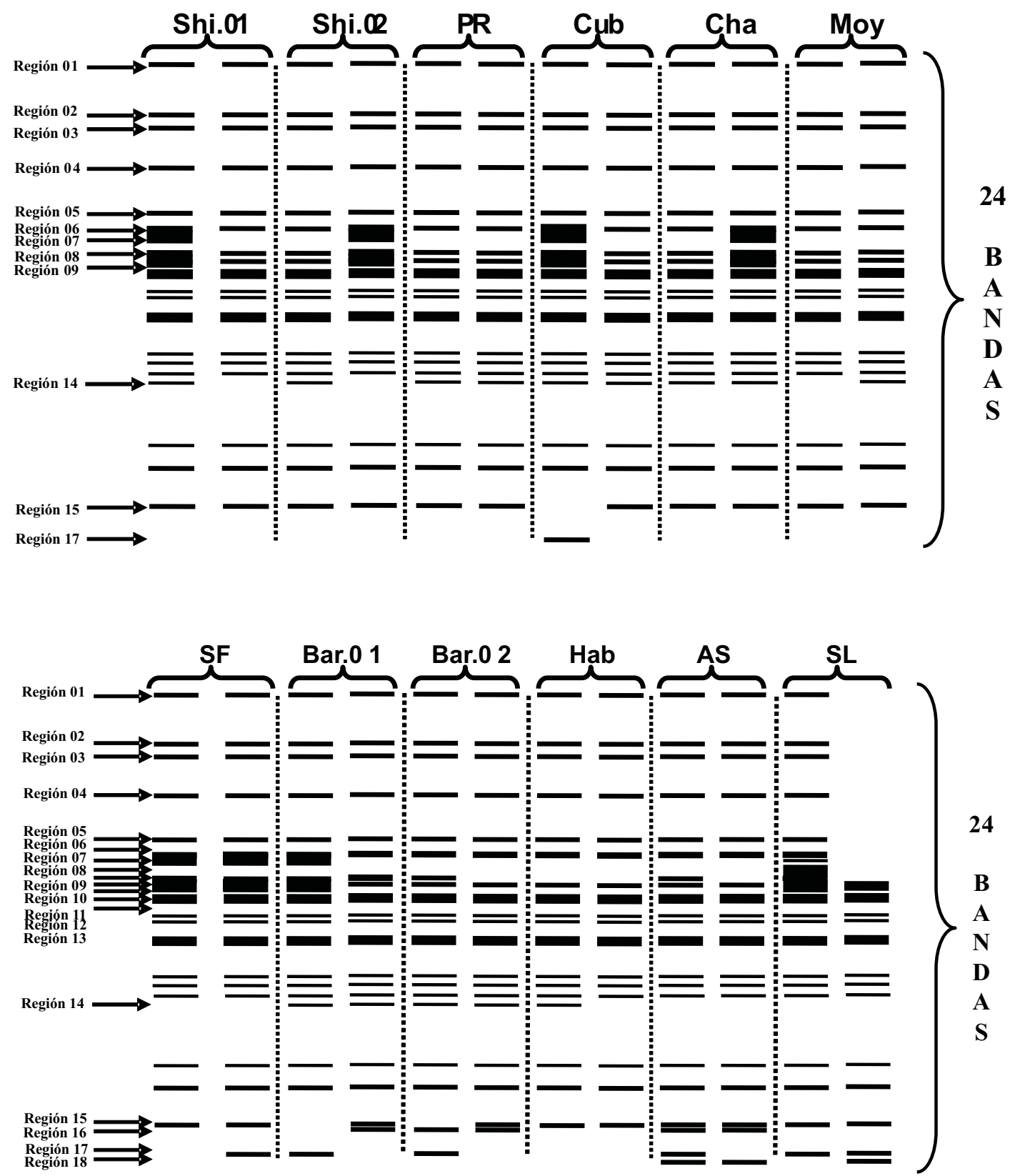

Figura 2. Representación gráfica de los geles de poliacrilamida $6 \%$ mostrando los fragmentos de ADN y las regiones informativas amplificadas con el marcador DALP233 en las accesiones de sacha inchi Plukenetia volubilis del Banco de Germoplasma del INIA. 


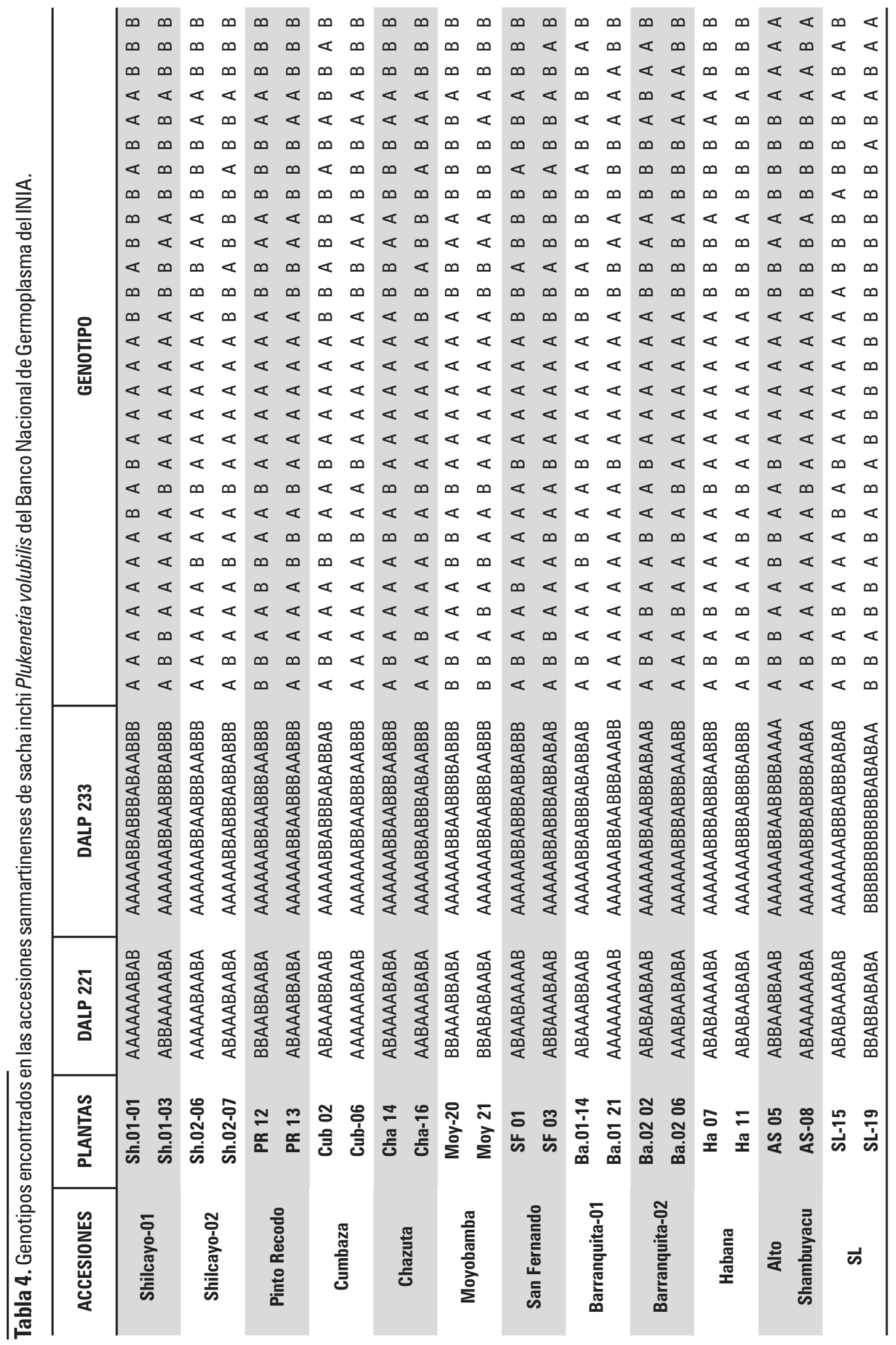


Tabla 3. Número y secuencia de los marcadores de la técnica DALP utilizados para caracterizar las accesiones de sacha inchi Plukenetia volubilis.

\begin{tabular}{ccccc}
\hline $\begin{array}{c}\text { MARCADORES } \\
\text { POLIMÓRFICOS }\end{array}$ & $\begin{array}{c}\text { TOTAL DE BANDAS } \\
\text { AMPLIFICADAS }\end{array}$ & $\begin{array}{c}\text { BANDAS } \\
\text { INFORMATIVAS }\end{array}$ & PBI (\%) \\
\hline DALP221 & 22 & 18 & 39.13 \\
DALP233 & 24 & 10 & 21.74 \\
\hline TOTAL & $\mathbf{4 6}$ & $\mathbf{2 8}$ & $\mathbf{6 0 . 8 7}$ \\
\hline PROMEDIO & $\mathbf{2 3}$ & $\mathbf{1 4}$ & $\mathbf{3 0 . 4 4}$ \\
\hline
\end{tabular}

PBI: Porcentaje de bandas informativas.

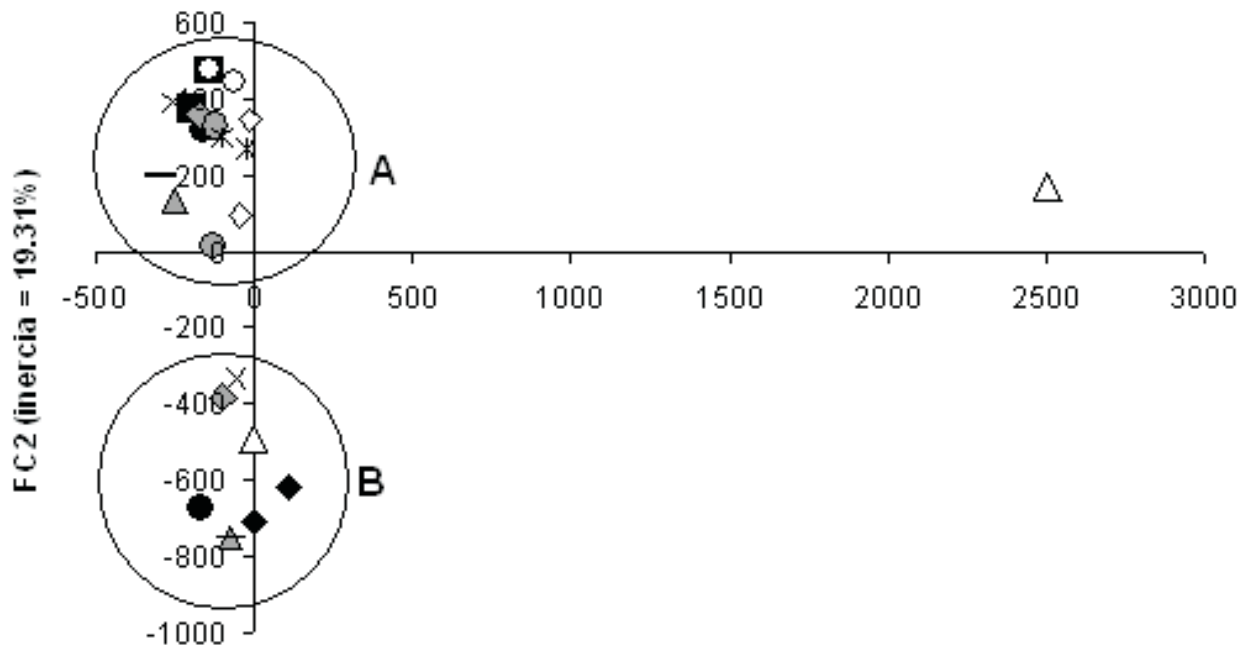

FC 1 (inercia $=28.09 \%$ )

$\begin{array}{llll}\text { - Shilcayo-01 } & \times \text { shilcayo-02 } & \text { Dinto Recodo } & \Delta \text { Cumbaza } \\ \diamond \text { Chazuta } & \text { OMoyobamba } & \text { \&an Fernando } & - \text { Barranquita-01 } \\ \text { obarranquita-02 } & \text { * Habana } & \diamond \text { Alto Shanbuyacu } & \Delta S L-1\end{array}$

Figura 3. Representación gráfica de los resultados del Análisis Factorial de Correspondencia (AFC), distribuidos sobre un plan factorial para los ejes 1 Vs. 2 para los individuos del Banco Nacional de Germoplasma de sacha inchi Plukenetia volubilis del INIA en función a sus genotipos. 


\section{AGRADECIMIENTO}

Al Proyecto Innovación y Competitividad para el Agro Peruano - INCAGRO, por el financiamiento parcial del presente estudio a través del subproyecto "Obtención de líneas mejoradas de sacha inchi (Plukenetia volubilis L.), a partir de material genético con altos rendimientos y contenidos de omega 3 y omega 6".

\section{BIBLIOGRAFÍA CITADA}

Belkhir, K.; Borsa, P.; Chichi, I.; Raufast, N.; Bonhomme, F. 2004. GENETIX 4.05.2, logiciel sous windowns TM pour la genétique des populations. Laboratoire génome, populations, interactions, CNRS UMR 5000, Université de Montpellier II, Montpellier, France.

Borém A.; Caixeta, E. 2006. Marcadores Moleculares. Editora Jard, Viçosa, 374p.

Corazon-Guivin, M.; Rodríguez, A.; Cachique, D.; Chota, W.; Vásquez, G.; Del-Castillo, D.; JeanFrançois R.; García-Dávila, C. 2008. Diversidad Genética en Poblaciones Naturales de Sacha Inchi plukenetia volubilis L. (euphorbiaceae) en el Departamento de San Martín (perú). Folia Amazónia. Vol. $17 \mathrm{~N}^{\circ}$ 1-2, p. $83-90$.

Desmarais, E.; Laneluc, I.; Lagnel, J. 1998. Direct amplification of length polymorphisms (DALP) or how to get and characterize new genetic markers in many species. Nucleic Acids Research. Vol. 26, 6:1458 1465 .

Doyle, J.J.; Doyle J.L. 1987. A rapid ADN isolation procedure for small quantities of fresh leaf tissue. Phytochem. Bull. Vol. 19:11-15.
Ferreira, M.Y.; Grattapaglia, D. 1998. Introducción al uso de marcadores moleculares en el análisis genético. EMBRAPA-CENARGEN, Brasilia, Brasil.p. 38-56

Ha, W.Y.; Yau, F.C.; But, P.P.; Wang, J.; Shaw, P.C. 2001. Direct amplification of length polymorphism analysis differentiates Panax ginseng from P. quinquefolius. Planta medica. Vol. 67(6):587-9.

Hamaker, B.R.; Valles, C.; Gilman, R.; Hardmeier, R. M.; Clark, D.; García, H.H.; Gonzales, A. E.; Kohlstad, I.; Castro, M. 1992. Amino Acid and Fatty Acid Profiles of the Inca Peanut (Plukenetia volubilis L.), Cereal Chem. Vol. 69:461-463.

Perrot-Minnot, M.J.; Lagnel, J.; Desmarais, E.; Navajas, M. 2000. Isolation and characterization by direct amplification of length polymorphism (DALP) of codominant genetic marker with mendelian inheritance in Neoseiulus californicus (Acari: Phytoseiidae). Exp. Appl. Acarol. Vol. 24: 795-803.

Rodríguez, A.; Corazon-Guivin, M.; Cachique, D.; Mejía, K.; Del Castillo, D.; Jean-François, R.; García-Dávila, C. 2010. Diferenciación morfológica y por ISSR (Inter simple sequence repeats) de especies del género Plukenetia (Euphorbiaceae) de la Amazonía peruana: propuesta de una nueva especie. Rev. peru biol., vol.17, no.3, p.325-330.

Rossiter, S.; Jones, G.; Ransome, R.; Barratt, E. 2000. Genetic variation and population structure in the endangered greater Rinolophus ferrumequinum. Molecular Ecology. Vol. 9:1131-1135. 